\title{
Expectativas del futuro profesorado de Educación Infantil y Primaria sobre el desarrollo infantil en distintas estructuras familiares ${ }^{1}$
}

\author{
Beatriz Triana Pérez, Esperanza Mª Ceballos Vacas y Juan A. Rodríguez Hernández
}

Universidad de La Laguna - España

\begin{abstract}
RESUMEN
Los prejuicios sociales del profesorado sobre las familias no convencionales pueden ser un escollo más en la relación familia-escuela. Por ello, se exploraron las expectativas de 220 estudiantes de los Grados en Maestro de Educación Infantil y Primaria sobre la adaptación de los hijos/as que crecen en distintas modalidades familiares. Se realizó un estudio cuantitativo de encuesta por muestreo incidental, con datos descriptivos y contrastes para pruebas no paramétricas. Las respuestas dadas a 19 ítems, usando una escala tipo Likert de 10 puntos, muestran una visión menos prejuiciosa hacia la diversidad. No obstante, la familia nuclear tradicional sigue recibiendo las expectativas de mayor adaptación. Le sigue la familia adoptiva biparental heterosexual y la condición de custodia compartida tras la ruptura. Las peores expectativas recaen en las familias reconstituidas, especialmente en presencia de una madrastra y sus hijos/as, o en los casos de familias monoparentales con custodias en solitario. En general, las mayores expectativas de adaptación se asocian a las condiciones de biparentalidad, heterosexualidad, existencia de vínculos biológicos con el cuidador principal (especialmente con la madre), y cuanto menor número de miembros nuevos se incorporen a la familia. Así, aunque los prejuicios sociales parecen matizarse, se ve necesaria una mayor formación del profesorado sobre los retos específicos de las distintas estructuras familiares evaluadas.
\end{abstract}

Palabras Clave: Familia, escuela, diversidad, expectativas, profesorado.

\section{Expectations of trainee infant and primary teachers on child development in different family structures}

\section{ABSTRACT}

The social prejudices of teachers regarding unconventional families can be another obstacle in the family-school relationship. For this reason, the expectations of 220 undergraduates taking teaching degrees on the adaptation of children growing up in different family modalities are explored. A quantitative survey with incidental sampling was carried out, presenting descriptive data and contrasts for non-parametric tests. Participants responded to 19 items using a 10-point Likert scale. The results show a less prejudiced view of diversity. However, the traditional nuclear family continues to receive expectations of better adaptation. It is followed by the heterosexual biparental adoptive family and the shared custody condition after a breakup. The worst expectations fall on blended families, especially in the presence of a stepmother and her children, or the case of single-parent families with sole custody. In general, the highest expectations of adaptation are associated with conditions of biparentality, heterosexuality, existence of biological links with the main caregiver (especially with the mother), and a low number of new members joining the family. Thus, although social prejudices seem to be moderated, it is necessary to provide more teacher training on the specific challenges of different family structures.

Keywords: Family, school, diversity, expectations, teachers.

\section{Marco teórico}

Existe un consenso generalizado acerca de los beneficios de la adecuada relación familia-escuela que repercuten tanto en el profesorado como en el alumnado y sus familias (Gomariz, Hernández-Prado y Parra, 2017; Oliva y Palacios, 2005). A pesar de estas bondades, el diagnóstico de la relación de la familia con la escuela, en nuestro país, es francamente mejorable (Ministerio de Educación, Cultura y Deporte, 2014). La escasa relación entre ambos agentes de socialización admite varias razones: desajustes en los horarios, desinterés de las familias, ausencia de iniciativa por parte de la escuela, entre otras, aunque una de las más obvias es la percepción negativa mutua entre las familias y el profesorado. Una primera clave para evitar este desencuentro es

Trabajo subvencionado con el proyecto del Ministerio de Economía y Competitividad, Ref. EDU2012-38588. 
promover la comunicación entre ambos en aras de un mejor conocimiento y comprensión mutuas (Muller, 2006).

La escuela, como institución educativa, está abocada a asumir la responsabilidad de iniciar este acercamiento (Rodríguez-Ruiz, Martínez-González y Rodrigo, 2016). Esto implica, en palabras de Gomariz, Hernández-Prados, García-Sanz y Parra (2017; p.44), "abrir las posibilidades de inclusión y participación familiar en las cuestiones escolares, partiendo de un estudio pormenorizado de la realidad familiar y adaptando las vías de participación...". Para lograr este desafío, al profesorado de Educación Infantil y Primaria se le presume pertrechado con un bagaje de conocimiento acerca de las características y procesos familiares. Sin duda, esta preparación es decisiva a la hora de comprender las necesidades del alumnado y de sus familias.

En España, de acuerdo con las cifras del Instituto Nacional de Estadística (INE, 2018), existe una creciente variedad de configuraciones familiares. Aunque la familia nuclear sigue siendo la más representativa, ésta comparte espacio con otras fórmulas familiares biparentales como las adoptivas, y con otras aún más novedosas, y en alza, como las reconstituidas y homoparentales. Prácticamente, uno de cada cuatro hogares de familias con descendencia es monoparental (83\% a cargo de la madre), ya sea por viudedad, divorcio o separación, o como opción en solitario a la maternidad/paternidad. Además, pueden constituirse con hijos e hijas biológicos y/o adoptivos, y encabezarse por una persona adulta heterosexual u homosexual. Conseguir datos precisos de algunos modelos familiares resulta complicado, por no registrarse oficialmente todos los casos, como en la reconstitución (Pryor, 2014), o por el ocultismo que perdura en España respecto a la orientación homosexual, en la homoparentalidad (Cortina, 2016).

Otros datos merecen mención en el análisis, retratando así el panorama nacional, y más específicamente, el de la Comunidad Autónoma de Canarias: Es una de las regiones con mayor porcentaje de rupturas legales en España, donde el 40,7\% tiene descendencia menor de edad; el 23,4\% de los divorcios y el 14,9\% de las separaciones se produce por vía contenciosa; y se aprecia un cierto avance hacia la custodia compartida, aunque en menor grado que en otros puntos de España, primando la custodia de las madres (Rodrigo, 2018). Ello favorece el fortalecimiento de la relación con sus vástagos y la preeminencia de su rol ante la sociedad, en detrimento de los padres (Baum, 2004; Braver, Ellman y Fabricius, 2003). Como es de suponer, el incremento de rupturas lleva aparejado también un aumento de reconstituciones.

Podemos asumir entonces que un porcentaje nada desdeñable de menores sufren, antes, durante y/o tras la ruptura, o también, tras la reconstitución, el estrés del conflicto y de los tránsitos familiares. Ello limita su bienestar, pudiendo presentar dificultades de adaptación de diversa índole, como la presencia de problemas externalizantes de conducta (agresión, desobediencia...) e internalizantes (depresión, baja autoestima...), dificultades de socialización, y también problemas académicos (menor rendimiento, mayor índice de abandono...), en comparación con los hijos/as de familias nucleares (Hetherington y Kelly, 2005; Justicia y Cantón, 2007; Pryor, 2014). Sin embargo, la gran mayoría reduce estas manifestaciones con el tiempo, y tan sólo cuando perdura el conflicto tras la ruptura, se mantienen o acrecientan dichos efectos (Golombok, 2006; Strohschein, 2005).

Por otra parte, y como ha ocurrido en el resto de España, la adopción ha sido una práctica común en Canarias, tanto en su modalidad internacional como nacional, aunque se ha apreciado una considerable reducción tras la crisis económica de los últimos años (odrigo, 2018). Así, se observa en la sociedad una gran heterogeneidad de configuraciones familiares, entre las que participan también las homoparentales. Todas las familias adoptivas se enfrentan a la tarea de acompañar a sus hijos/as en la elaboración de su historia e identidad. Además, y tras la reciente crisis económica y las mayores restricciones impuestas por algunos países, los retos y esfuerzos de las familias son más complejos al ser más común la adopción de menores con características especiales (pe., algún tipo de discapacidad o enfermedad; inadaptación social relevante; ocho o más años; y/o grupos de varios hermanos/as), lo que se acompaña de mayores necesidades a las que responder. No obstante, no son la mayoría de los casos, y además, sus conductas extremas tampoco son siempre clínicamente significativas en comparación con lo observado en las familias no adoptivas (Berástegui, 2005; Palacios y Sánchez, 1996; Sagarna, 2010).

En paralelo, el notorio incremento de uniones entre hombres, y aún más, de mujeres, suscita un mayor debate social sobre los posibles efectos de crecer en familias homoparentales frente a tradicionales, si bien la investigación, en general, muestra mínimas diferencias al respecto. Como señalan Brabender y Fallon (2013), en una amplia revisión de estudios, los problemas se derivan principalmente de los prejuicios externos a la familia, ya que su dinámica familiar y la atención hacia los hijos e hijas es similar a la observada en las familias tradicionales. Incluso, con eventuales beneficios como la mayor aceptación de las diferencias (Farr y Patterson, 2013).

Una vez descrito brevemente este panorama social de las familias españolas, cabe preguntarse si el profesorado es consciente de esta diversidad familiar, y cuál es su apreciación de dichas estructuras familiares. De acuerdo con Morgado, Jiménez-Lagares y González (2009), los estudios muestran creencias sesgadas ante la diversidad familiar, en las que la tendencia hacia la hetero-normatividad y la homofobia desempeñan un papel relevante. Esta visión del profesorado cobró protagonismo a partir de los años 80, en la literatura anglosajona, asociando expectativas más negativas al alumnado proveniente de familias no tradicionales. Por ejemplo, en las familias monoparentales por divorcio (Ball, Newman y Scheuren, 1984) y en las familias homoparentales respecto a su adaptación social (Bliss y Harris, 1999). También, Guttmann, Geva y Gefen (1988) observaron en el profesorado un sesgo de atribución de peor funcionamiento académico, emocional y social al alumnado, cuando se les informaba de que su padre y su madre estaban divorciados.

En España, Morgado et al., (2009) encontraron tendencias similares en el profesorado andaluz de educación primaria, especialmente en el de mayor edad, quienes juzgaron las familias biparentales heterosexuales (adoptivas o no) como el contexto más favorable para el desarrollo infantil, preocupándoles el impacto del divorcio, y considerando más perjudicial la ausencia de la madre, frente a la del padre. También aventuraron efectos negativos en las familias homoparentales. Entendemos que estas creencias son difíciles de cambiar, y que se darán de forma análoga en las restantes comunidades españolas. Por tanto, nuestro estudio espera confirmar aún hoy, y en una muestra canaria, la supremacía en la valoración de las familias biparentales heterosexuales frente a las restantes modalidades.

Fredericks, Rasinski y Ritty (1991) advertían del peligro de la transmisión de valoraciones negativas del profesorado, tanto de forma directa como indirecta, sobre la idoneidad de las familias no convencionales. Asumir dichas expectativas puede provocar en el alumnado una menor autoconfianza, y una merma real en el rendimiento académico y la disciplina escolar, como efecto de una profecía autocumplida. Además, negar la pluralidad familiar y/o asumir estos prejuicios, añade nuevos obstáculos a la relación familia-escuela, con repercusiones discriminatorias para 
muchas familias (Rodríguez-Mena y Moreno, 2017). Esta resistencia de la escuela ante la complejidad familiar ha sido criticada desde la perspectiva inclusiva (Aguado, 2010), argumentando la falta de formación del profesorado y sus disfunciones sobre diversidad afectivo-sexual (Ceballos, 2009).

Esta subjetividad no solo afecta a la profesión educativa. También impregna la investigación en forma de sesgos (González, 2009) por asumir modelos teóricos de déficit o patógenos, centrados principalmente en los problemas y no en los beneficios; y la presencia de defectos en los diseños y la interpretación de los resultados, obviando variables moduladoras como el estatus socioeconómico, educativo y ocupacional, o las prácticas educativas familiares (Cantón y Justicia, 2007; Marí-Klose y Marí-Klose, 2010). Sin embargo, es factible suponer que el profesorado más joven matice el peso de estos prejuicios sociales. Por ejemplo, en estudios con profesorado en formación de otros países se apunta una mayor tolerancia hacia la homoparentalidad, especialmente hacia las parejas de lesbianas (Averett y Hedge, 2012). Aún así, persisten actitudes más positivas hacia las parejas heterosexuales (Herbstrith, Tobin, Hesson-McInnis y Schneider, 2013).

No tenemos constancia de la existencia de investigaciones con profesorado en formación en España, salvo el realizado por Abril y Peinado (2018) con futuro profesorado de la etapa Infantil, quienes encuentran actitudes más favorables hacia la diversidad familiar, pero aún con ciertas reticencias. De ahí nuestro interés en desarrollar un estudio con alumnado de los grados de Maestro/a de Educación Infantil y Primaria. Concretamente, se pretende analizar qué modelos familiares reciben actualmente una percepción más prejuiciosa respecto a la adaptación de los hijos e hijas que crecen en ellos. Un segundo objetivo es comprobar si diferentes estructuras, dentro de cada modalidad, afectan a los resultados obtenidos. Así, y de acuerdo con los resultados previos, se prevén expectativas de mayor adaptación infantil respecto a la familia nuclear tradicional frente al resto de modalidades evaluadas. También se espera encontrar una mejor valoración para las estructuras biparentales, las encabezadas por una persona o una pareja heterosexual, aquéllas donde la madre esté presente en el hogar, o existan lazos biológicos parento-filiales.

\section{Método}

\subsection{Participantes}

En el estudio participaron 220 estudiantes (72,7\% mujeres y $27,3 \%$ varones) de los títulos de Maestro/a en la Universidad de La Laguna (42,3\% de Educación Infantil y 57,7\% de Educación Primaria). El muestreo se realizó de modo incidental entre alumnado de ambos grados, de los distintos cursos. La media de edad de los participantes es de 21,03 años y la desviación típica 3,7.

\subsection{Instrumento}

Se administró un instrumento confeccionado ad hoc, conformado por 15 apartados con preguntas abiertas y cerradas, para expresar las expectativas y experiencias sobre diferentes tipos de familia. Las cuestiones se seleccionaron tras la revisión de documentos como los de Brabender y Fallon (2013), Cantón, Cortés y Justicia (2007), Erera (2002), Golombok (2006, 2015), Loizaga (2010) o Pryor (2014), entre otros; y partiendo además de las sugerencias de dos profesionales especialistas en diversidad familiar. Para este trabajo se han recogido solo las respuestas a 19 cuestiones en las que se pregunta: "En qué grado consideras que se adaptan (es decir, que se desarrollan sin problemas) los hijos/as que crecen en las distintas modalidades familiares que se te presentan a con- tinuación". Seguidamente, se muestran las diferentes tipologías (en ítems diferenciados según la composición específica de sus miembros) de cada modalidad familiar (familias adoptivas y homoparentales, divorciadas, reconstituidas, y nuclear). Por ejemplo, algunos ítems relacionados con el modelo de familia reconstituida son: cuando los hijos/as viven habitualmente con su madre y su padrastro; cuando los hijos/as viven habitualmente con su padre, su madrastra y los hijos/as de esta última...). Para la valoración se usó una escala tipo Likert de 10 puntos (0-Nada; 10-Totalmente). La modalidad nuclear se representa con un ítem, y las restantes no convencionales a través de diferentes estructuras que las representan, seleccionándose las más comunes:

1. Adoptivas heterosexuales (3 ítems): monoparental con madre, monoparental con padre, biparental.

2. Adoptivas homoparentales (6 ítems): monoparental con madre lesbiana, monoparental con padre gay, biparental con pareja de madres lesbianas, biparental con pareja de padres gays, biparental con pareja de lesbianas (siendo una la madre biológica) y biparental con pareja de gays (siendo uno el padre biológico).

3. Monoparentales tras la separación según el tipo de custodia (3 ítems): madre custodia, padre custodio, custodia compartida.

4. Reconstituidas (6 ítems): con madre y padrastro; padre y madrastra; madre, padrastro y los hijos/as de él; padre, madrastra y los hijos/as de ella; madre y padrastro e hijo/a común; y padre y madrastra e hijo/a común.

\subsection{Procedimiento}

Se contactó con el profesorado de los distintos cursos en los Grados de Maestro para solicitar la colaboración en el estudio, intentando que la participación fuera representativa de la población de referencia, en número y género del alumnado. El alumnado participó de forma voluntaria (garantizándose el anonimato), durante las clases y en el periodo lectivo. El tiempo empleado para completar el cuestionario osciló entre 30 y 40 minutos.

\subsection{Análisis de datos}

Se trata de un estudio cuantitativo de encuesta. Los análisis se realizaron sobre 19 ítems, y han consistido en: a) datos descriptivos, medias $(M)$ y desviaciones típicas $(D T)$ para cada estructura; y b) contrastes de muestras relacionadas para pruebas no paramétricas, mediante la t de Wilcoxon, y el grado del efecto $(r)$ de dichos contrastes entre las distintas estructuras exploradas.

\section{Resultados}

Los descriptivos y los valores de los contrastes relativos a las distintas estructuras analizadas, para las modalidades de adopción y de reconstitución, se presentan en las tablas 1 y 2, respectivamente. Los resultados muestran que, en términos generales, todas las fórmulas familiares son aceptadas por los participantes como contextos válidos para la infancia, con promedios aproximados al 5 o superiores. De acuerdo con lo esperado, continúa la percepción de supremacía de la familia nuclear, que obtiene la expectativa de adaptación infantil más elevada $(M=9,21)$ y con mayor consenso $(D T=1,15)$.

En este sentido, se observan diferencias significativas de la modalidad nuclear frente a las distintas estructuras adoptivas exploradas: con pareja adoptiva heterosexual $(z=-5,70 ; p<, 000 ; r=$ ,38); madre heterosexual $(z=-9,81 ; p<, 000 ; r=, 66)$; pareja de ma- 
dres lesbianas $(z=-9,92 ; p<, 000 ; r=, 67$,$) ; padre heterosexual (z=$ $-10,26 ; p<, 000 ; r=, 69)$; pareja gay $(z=-10,11 ; p<, 000 ; r=, 68)$; madre lesbiana $(z=-10,82 ; p<, 000 ; r=, 73)$; padre gay $(z=-10,93 ; p<, 000 ; r=$ ,73); pareja de madres lesbianas, siendo una madre biológica $(z=$ $-10,94 ; p<, 000 ; \mathrm{r}=, 73) ; \mathrm{y}$ pareja gay, siendo uno padre biológico $(z=-11,12 ; p<, 000 ; r=, 75)$. En todos los casos, el tamaño del efecto de las diferencias significativas es grande, excepto el contraste con pareja adoptiva heterosexual, que es mediano.

Centrándonos ahora en el análisis de las diferentes configuraciones adoptivas (ver Tabla 1), la estructura biparental heterosexual emerge como la siguiente más valorada, tras la nuclear, con grados del efecto grandes. Con diferencias significativas se encuentran después las fórmulas adoptivas compuestas solo por mujeres como progenitoras, ya sea en su versión de madre adoptiva heterosexual o pareja de lesbianas, respecto a las restantes configuraciones, salvo en el caso de la pareja de lesbianas que no se diferencia significativamente del padre heterosexual. En estos casos, los grados del efecto observados son mayoritariamente medianos y grandes, salvo los contrastes de madre heterosexual con pareja de padres gay, o pareja de madres lesbianas con pareja gay y con madres lesbianas cuando una guarda vínculo biológico, en los que el efecto es pequeño. Le siguen en predicción de adaptación las conformadas solo por hombres en su versión de padre adoptivo heterosexual o la pareja gay, con grados del efecto pequeños y medianos. A continuación, aparecen las estructuras monoparentales con madre lesbiana o con padre gay, y pareja de madres lesbianas y pareja de padres gay donde un miembro tiene vinculación biológica con el hijo/a. En estas dos últimas la expectativa de menor adaptación recae en la pareja gay, con un efecto mediano.

Tabla 1.

Medias (M) y desviaciones típicas (DT), y diferencias del grado de adaptación infantil, en las distintas estructuras familiares adoptivas

\begin{tabular}{|c|c|c|c|}
\hline Estructura explorada $M(D T)$ & Estructura de contraste & $z$ & $r$ \\
\hline \multirow{8}{*}{$\begin{array}{l}\text { Padre y madre heterosexuales } \\
\qquad 8,59(1,78)\end{array}$} & madre heterosexual & $-8,02^{* * *}$ &, 54 \\
\hline & dos madres lesbianas & $-7,88^{* * *}$ & ,53 \\
\hline & padre heterosexual & $-8,79^{* * *}$ &, 59 \\
\hline & pareja padres gays & $-8,23^{* * *}$ &, 55 \\
\hline & madre lesbiana & $-9,29 * * *$ & ,62 \\
\hline & padre gay & $-9,63^{* * *}$ & 65 \\
\hline & pareja madres lesbianas (una madre biológica) & $-9,00^{* * *}$ & 60 \\
\hline & pareja padres gays (uno padre biológico) & $-9,46^{* * *}$ & 63 \\
\hline \multirow{7}{*}{$\begin{array}{c}\text { Madre heterosexual } \\
7,74(1,92)\end{array}$} & pareja madres lesbianas & n.s. & - \\
\hline & padre heterosexual & $-4,16^{* * *}$ & ,32 \\
\hline & pareja padres gays & $-3,12^{* *}$ &, 21 \\
\hline & madre lesbiana & $-7,08^{* * *}$ &, 51 \\
\hline & padre gay & $-7,18^{* * *}$ &, 50 \\
\hline & pareja madres lesbianas (una madre biológica) & $-5,17^{* * *}$ & ,35 \\
\hline & pareja padres gays (uno padre biológico) & $-6,13^{* * *}$ & 41 \\
\hline \multirow{6}{*}{$\begin{array}{c}\text { Pareja madres lesbianas } \\
7,53(2,10)\end{array}$} & padre heterosexual & n.s. & - \\
\hline & pareja padres gays & $-3,04^{* *}$ & 20 \\
\hline & madre lesbiana & $-5,31^{* * *}$ &, 36 \\
\hline & padre gay & $-5,52^{* * *}$ & ,37 \\
\hline & pareja madres lesbianas (una madre biológica) & $-3,59^{* * *}$ &, 24 \\
\hline & pareja padres gays (uno padre biológico) & $-4,81^{* * *}$ & ,32 \\
\hline \multirow{5}{*}{$\begin{array}{c}\text { Padre heterosexual } \\
7,49(2,01)\end{array}$} & pareja padres gays & n.s. & - \\
\hline & madre lesbiana & $-4,65^{* * *}$ & ,31 \\
\hline & padre gay & $-4,96^{* * *}$ & ,33 \\
\hline & pareja madres lesbianas (una madre biológica) & $-3,30^{* *}$ & ,22 \\
\hline & pareja padres gays (uno padre biológico) & $-4,39^{* * *}$ & ,31 \\
\hline \multirow{4}{*}{$\begin{array}{c}\text { Pareja padres gays } \\
\quad 7,37(2,26)\end{array}$} & madre lesbiana & $-3,20^{* *}$ &, 21 \\
\hline & padre gay & $-3,60^{* * *}$ &, 24 \\
\hline & pareja madres lesbianas (una madre biológica) & $-2,10^{*}$ & ,14 \\
\hline & pareja padres gays (uno padre biológico) & $-3,05^{* *}$ & .23 \\
\hline \multirow{3}{*}{$\begin{array}{l}\text { Madre lesbiana } \\
\quad 7,08(2,17)\end{array}$} & padre gay & n.s. & - \\
\hline & pareja madres lesbianas (una madre biológica) & n.s. & - \\
\hline & pareja padres gays (uno padre biológico) & n.s. & - \\
\hline
\end{tabular}




\begin{tabular}{clcl}
\hline & & & \\
\hline Padre gay & pareja madres lesbianas (una madre biológica) & n.s. \\
pareja padres gays (un padre biológico) & n.s. & - \\
\hline $\begin{array}{c}\text { Pareja madres lesbianas (una } \\
\text { madre bióógica) } \\
7,05(2,24)\end{array}$ & pareja padres gays (un padre biológico) & $-4,09^{* * *}$ & \\
\hline $\begin{array}{c}\text { Pareja padres gays (un padre } \\
\text { biológico) } \\
6,90(2,23)\end{array}$ & & \\
\hline
\end{tabular}

Nota: n.s. $=$ No significativo ${ }^{*} \mathrm{p}<.05 ;{ }^{* *} \mathrm{p}<0,01 ;{ }^{* * *} \mathrm{p}<0,001$. Grado del Efecto: pequeño $<.3 ;$ mediano $\geq .3$ y $<.5 ;$ grande $\geq .5$

Siguiendo con el análisis de las expectativas de adaptación infantil, tras el valor obtenido por la estructura adoptiva biparental heterosexual, se observa como la siguiente más favorable, la monoparental con custodia compartida $(z=-3,00 ; p<, 01 ; r=, 20)$, con un grado del efecto pequeño. Por otra parte, en los casos explorados de separación/divorcio, las expectativas son significativamente mejores $(z=-7,97 ; p<, 000 ; r=, 54)$ en la situación de custodia com- partida $(M=8,18 ; D T=1,89)$ frente a la custodia exclusiva de la madre $(M=6,61 ; D T=1,97)$, y también del padre $(M=6,13 ; D T=1,85)$ $(z=-9,76 ; p<, 000 ; r=, 66)$. Estos resultados se asocian con efectos grandes. Además, de acuerdo con la preferencia esperada por la figura femenina, se obtiene una expectativa de mayor adaptación en la custodia exclusiva de la madre frente a la del padre $(z=-5,97$; $p<, 000 ; r=, 40)$, con un grado del efecto moderado.

Tabla 2.

Medias (M) y desviaciones típicas (DT), y diferencias del grado de adaptación infantil, en las distintas estructuras familiares reconstituidas

\begin{tabular}{|c|c|c|c|}
\hline Estructura explorada $M(D T)$ & Estructura de contraste & $z$ & $r$ \\
\hline \multirow{5}{*}{$\begin{array}{l}\text { Con padrastro } \\
6,37(1,94)\end{array}$} & con madrastra & $-6,00^{* * *}$ & 40 \\
\hline & con padrastro y nuevo hijo/a común & $-4,35^{* * *}$ & ,30 \\
\hline & con madrastra y nuevo hijo/a común & $-5,40^{* * *}$ & 36 \\
\hline & con padrastro y sus hijos/as & $-9,64^{* * *}$ & 65 \\
\hline & con madrastra y sus hijos/as & $-9,53^{* * *}$ & 64 \\
\hline \multirow{4}{*}{$\begin{array}{l}\text { Con madrastra } \\
5,81(1,84)\end{array}$} & con padrastro y nuevo hijo/a común & n.s. & - \\
\hline & con madrastra y nuevo hijo/a común & $-2,43^{*}$ & , 16 \\
\hline & con padrastro y sus hijos/as & $-5,58^{* * *}$ & ,37 \\
\hline & con madrastra y sus hijos/as & $-7,78^{* * *}$ &, 52 \\
\hline \multirow{3}{*}{$\begin{array}{l}\text { Con padrastro y nuevo hijo/a común } \\
\qquad 5,77(2,15)\end{array}$} & con madrastra y nuevo hijo/a común & $-3,69^{* * *}$ & 25 \\
\hline & con padrastro y sus hijos/as & $-4,46^{* * *}$ &, 31 \\
\hline & con madrastra y sus hijos/as & $-5,68^{* * *}$ & ,38 \\
\hline \multirow{2}{*}{$\begin{array}{l}\text { Con madrastra y nuevo hijo/a común } \\
\qquad 5,52(2,18)\end{array}$} & con padrastro y sus hijos/as & $-2,63^{* *}$ & , 18 \\
\hline & con madrastra y sus hijos/as & $-4,39^{* * *}$ & 30 \\
\hline $\begin{array}{l}\text { Con padrastro y sus hijos/as } \\
\qquad 5,18(1,97)\end{array}$ & con madrastra y sus hijos/as & $-3,01^{* *}$ & ,20 \\
\hline $\begin{array}{l}\text { Con madrastra y sus hijos/as } \\
\qquad 4,95(2,00)\end{array}$ & & & \\
\hline
\end{tabular}

Nota: n.s.= n.s. $=$ No significativo ${ }^{*} \mathrm{p}<.05 ;{ }^{* *} \mathrm{p}<0,01 ;{ }^{* * *} \mathrm{p}<0,001$. Grado del Efecto: pequeño $<.3$; mediano $\geq .3 \mathrm{y}<.5 ;$ grande.$\geq 5$

Las familias reconstituidas alcanzan las expectativas de adaptación infantil más bajas y alejadas de las familias nucleares (ver Tabla 2). En este sentido, se observan diferencias significativas, con grado del efecto grande, al contrastar la modalidad nuclear frente a las distintas estructuras reconstituidas exploradas: con padrastro $(z=-11,95 ; p<, 000 ; r=, 81)$; madrastra $(z=-12,50 ; p<, 000$; $r=, 84)$; padrastro y nuevo hijo/a en común $(z=-12,27 ; p<, 000 ; r=$ ,83); madrastra y nuevo hijo/a en común $(z=-12,40 ; p<, 000 ; r=, 84)$; padrastro y sus hijos/as $(z=-12,67 ; p<, 000 ; r=, 85)$; y madrastra $y$ sus hijos/as $(z=-12,66 ; p<, 000 ; r=, 85)$.

Entre las familias reconstituidas analizadas, la estructura con padrastro obtiene el mayor valor de adaptación infantil, a dife- rencia de las restantes, y similar a las dos modalidades de custodia en solitario, con grados del efecto grandes y medianos. Le sigue en orden decreciente la reconstitución con madrastra, con padrastro y nuevo hijo/a común, y con madrastra y nuevo hijo/a en común. En estos casos los efectos son principalmente medianos y uno grande, salvo lo observado entre la estructura con madrastra, con madrastra y nuevo hijo/a en común; esta última, con padrastro y nuevo hijo/a en común; y con madrastra y nuevo hijo/a en común, con padrastro y sus hijos/as, donde los efectos son pequeños. A continuación, encontramos la conformada por el padrastro y sus hijos/as y, por último, la de la madrastra y sus hijos, con un tamaño de efecto pequeño. 


\section{Discusión}

En las últimas décadas, y tras los numerosos cambios legales, económicos, culturales y sociales acaecidos en España, el concepto de familia ha ido dando cabida a nuevas modalidades familiares, conformándose así el concepto actual de diversidad familiar (Erera, 2002). Ahora, ser familia ya no implica la necesidad de matrimonio, tener hijos/as, o poseer vínculos biológicos con la descendencia. Tampoco requiere que el hogar esté encabezado por dos personas adultas, que sean heterosexuales, o que compartan un mismo origen cultural. Así, las distintas modalidades familiares se hacen cada vez más visibles, construyendo una realidad social que se presenta, cuanto menos, compleja y heterogénea. No obstante, el conocimiento sobre las dinámicas y los retos de las diferentes fórmulas familiares está aún poco difundido, lo que ha propiciado la asunción de prejuicios sobre la capacidad de las familias no convencionales para la paternidad/maternidad. Estos mitos, aún muy arraigados en la sociedad, limitan la plena aceptación de algunos modelos familiares pues, obviamente, resulta más fácil visibilizarlos que normalizarlos (Abril y Peinado, 2018). La escuela cobra especial relevancia para impulsar dicho cambio. Sin embargo, el profesorado, al igual que otros agentes sociales, se apoya frecuentemente en esos mitos y prejuicios para valorar a las familias alternativas y formular predicciones sobre sus hijos, que no siempre se ajustan a la realidad (Bliss y Harris, 1999).

En este sentido se ha planteado el primer objetivo de este estudio que consiste en explorar las expectativas del estudiantado actual de los títulos de Maestro respecto a la adaptación infantil en diferentes familias. Los resultados confirman que, en general, los maestros en formación muestran posturas moderadas, y una visión menos prejuiciosa, en comparación con lo observado en estudios previos (Maney y Cain, 1997). Así, todas las fórmulas familiares son aceptadas como contextos válidos para la infancia, lo que indicaría que los estereotipos se están matizando, de acuerdo con lo que habíamos esperado. No obstante, prevén más problemas en el alumnado procedente de las familias reconstituidas, seguidas de las separadas/divorciadas con custodias exclusivas, y algo menos en las familias establecidas por adopción, especialmente en las homoparentales y la encabezada por un padre heterosexual. Este resultado más favorable asociado a la homoparentalidad también lo observaron Averett y Hedge (2012) en docentes en formación, y Morgado et al., (2009) en el profesorado en activo más joven, en comparación con el de mayor edad. La mayor familiaridad y contacto de estas poblaciones con personas homosexuales y/o familias homoparentales, especialmente en su entorno cercano, puede haber contribuido al cambio de percepción (Toro-Alfonso y Varas-Díaz, 2004).

Estos resultados suponen una noticia positiva porque indicarían una progresiva aceptación de la ineludible diversidad familiar (García-Pérez et al., 2011). No en vano, esta obligación se reconoce en los principios y fines de la educación desde la LOE (Ley Orgánica 2/2006, de Educación de 3 de mayo) y la LOMCE (Ley Orgánica 8/2013, para la Mejora de la Calidad Educativa de 9 de diciembre) que destacan, entre otros aspectos, la obligación de promover la equidad en la educación y la igualdad de oportunidades para todo el alumnado, evitando el trato discriminatorio; y la necesidad de impulsar, además, la tolerancia y el respeto hacia la diversidad. Por otra parte, las órdenes ECI/3854/2007 y ECI/3857/2007, de 27 de diciembre, que establecen los requisitos para la profesión de Maestro en Educación Infantil y Primaria, respectivamente, destacan como competencias la atención al alumnado y sus familias, respondiendo a sus necesidades educativas singulares.
Para el ejercicio de dichas competencias, la escuela puede actuar desde varias vertientes: a) Recoger y visibilizar la diversidad familiar; b) trabajar como escuela inclusiva, para que el alumnado y sus familias se sientan aceptadas y valoradas positivamente, con independencia de su modelo familiar, encontrando en ella referentes de su propia realidad (Ceballos, 2009); c) coordinarse con las familias para dar coherencia a los procesos educativos y a los valores que se transmitan en ambos contextos, facilitando así el desarrollo personal, socio-emocional y cognitivo de su alumnado (Oliva y Palacios, 2005); y d) apoyar al alumnado y a las familias que enfrentan dificultades y tránsitos familiares relevantes, ayudándoles a entender de forma más objetiva determinadas realidades, e informándoles sobre fuentes de apoyo externas e internas que puedan mitigar sus problemas (Hayman, 2008).

Para que la escuela pueda cumplir adecuadamente con dichos cometidos deberá superar varios escollos. Primero, conseguir información de la familia que ayude a entender sus retos particulares que pueden explicar determinados comportamientos del alumnado. Esto no siempre es posible porque algunas familias lo evitan por miedo a que la escuela pueda estigmatizar a sus hijos como efecto de los prejuicios (Casper, Schultz y Wickens, 1992). Segundo, que la escuela y las familias reconozcan el beneficio de una actuación colaboradora y coherente entre ambos. Tercero, que el profesorado esté preparado para entender las realidades familiares de su alumnado, y se haya liberado, además, de prejuicios sin fundamento. Y cuarto, que el profesorado no transmita creencias y actitudes sesgadas y prejuiciosas al alumnado y/o a sus familias, y contribuir así a la verdadera inclusión de la diversidad familiar.

Haber nacido a las puertas del siglo XXI, pudiendo ver de forma más natural la diversidad, ha ayudado a mitigar los estereotipos de nuestros participantes y, posiblemente, a ajustar también su conocimiento al respecto. Por ejemplo, conocer casos de homoparentalidad con buena adaptación familiar favorece que se relativicen prejuicios sin base objetiva. También puede contribuir a ello la legalización del matrimonio de personas del mismo sexo, así como la visibilización de la coparentalidad homosexual a través de parejas famosas. Asimismo, la mayor incidencia actual de la ruptura familiar, de la reconstitución y de la adopción, cada una de ellas con su propia heterogeneidad y complejidad estructural, puede llevar a una óptica algo diferente a la de estudios anteriores. Por ejemplo, la familia adoptiva ha sido tradicionalmente objeto de una visión tan positiva como la familia nuclear (Scanzoni, 2004). Sin embargo, hoy se admite su gran heterogeneidad en cuanto a retos y resultados de adaptación.

Por todo ello, es necesario que el profesorado disponga de un mayor bagaje formativo acerca de los retos específicos que cada familia debe enfrentar, en función de su tipología. Por ejemplo, sus debilidades, sus fortalezas, las variables que imprimen efectos en sus dinámicas y afectan a su adaptación, etc. (Golombok, 2015). Las versiones muy pesimistas de su idoneidad, y también las muy optimistas, suponen visiones parciales de dichas familias. Al ignorar estas especificidades, la escuela podría desplegar acciones inadecuadas hacia ellas. Así, y aunque nuestros participantes, al igual que los del estudio de Abril y Peinado (2018), siguen considerando la familia nuclear tradicional como el mejor entorno para el desarrollo infantil, es necesario que asuman que tampoco está exenta de dificultades. Cada modalidad familiar tiene sus propios desafíos, acompañados de luces y sombras (Erera, 2002), si bien todas comparten también importantes similitudes (Golombok, 2015).

Precisamente, para un análisis más específico de las expectativas del profesorado en formación respecto a la adaptación de los hijos e hijas en familias diversas, se exploró, como se- 
gundo objetivo, cómo determinados aspectos relacionados con la complejidad de cada estructura familiar, podían afectar a sus apreciaciones. Para ello, se analizaron diferentes variantes de las familias no convencionales, como su condición biparental o monoparental, el sexo de los progenitores, su orientación sexual, y la existencia o no de vínculo sanguíneo con los hijos e hijas. Los datos, en primer lugar, ponen de manifiesto que la biparentalidad heterosexual se relaciona con la expectativa de mayor adaptación de la descendencia, al igual que lo observado por Herbstrith et al., (2013). Así, después de la familia nuclear, el modelo más idóneo es la familia adoptiva heteroparental, seguido por la situación de custodia compartida donde ambos progenitores atienden las necesidades de sus hijos e hijas desde hogares diferentes. Probablemente, el no disponer de un único hogar que facilite la estabilidad emocional y rutina cotidiana, en el caso de la ruptura, lleve a percibir de forma algo más positiva a la familia adoptiva heteroparental.

A continuación, le siguen en orden decreciente las restantes estructuras adoptivas monoparentales heterosexuales, o también las conformadas por parejas lesbianas o gays, percibiéndose entre estas fórmulas un mayor ajuste en la familia con una madre heterosexual y en la biparental lesbiana, en comparación con la biparental gay, aunque dichas diferencias lo son a modo de tendencia. En menor grado, aunque aún con valores claramente moderados, aparecen las restantes estructuras adoptivas encabezadas por la monoparentalidad homosexual y las parejas homosexuales con vínculo biológico con uno de los adultos. Curiosamente, cuando se da dicho vínculo biológico, se espera mayor adaptación en las parejas de lesbianas que en las de gays. En este sentido, atender a la vinculación con los hijos e hijas por vía legal o biológica, genera predicciones diferenciadas sobre las relaciones con ellos (Mallon, 2000).

Por último, en las familias reconstituidas los datos indican expectativas de más adaptación cuando en el hogar principal reside la madre, probablemente por la atribución tradicional de una mayor capacidad como cuidadora, y por la ausencia de las madrastras o de hermanastros/as (figuras menos aceptada); cuando se tienen nuevos hijos en común, quizás porque el nuevo hermano/a facilitaría la vinculación de los hijos/as del progenitor con su nueva pareja; y en las estructuras menos complejas, probablemente porque habrá menos subsistemas de relaciones que afecten a la dinámica global. Estos resultados coinciden, en general, con algunos estudios que analizan las dinámicas de las familias reconstituidas (Ganong y Coleman, 2004; Pryor, 2014).

La convivencia con los padrastros se puede percibir más positiva que con las madrastras porque suelen asumir roles secundarios (de amigo y mediador) de los hijos de sus parejas, mientras que las madrastras se implican más en su crianza y educación, pudiendo generar rechazo en los hijastros. Al margen de estos eventuales conflictos, para explicar por qué el modelo de la madrastra que aporta hijos propios a la convivencia familiar recibe la evaluación más negativa de todas, es inevitable recordar la figura absolutamente peyorativa de la madrastra en el mito de la Cenicienta.

Por otro lado, tras la ruptura de la pareja, las familias encabezadas por madres se perciben más favorecedoras de la adaptación de los hijos/as, de nuevo en sintonía con el estereotipo de la mujer como cuidadora universal. No obstante, se aprecia un importante avance en el reconocimiento del papel de los varones en el cuidado de sus hijos/as, reduciendo así la división de roles sexista en la familia (Bjorklund y Jordan, 2013; Morgado et al., 2009). De cualquier modo, algunos estudios afirman que los niños que crecen en familias monoparentales, tras una ruptura, $y$ en las reconstituidas, son los que corren más riesgo (Golombok, 2015). Esta visión se refleja en las expectativas de los participan- tes del presente estudio, coincidiendo con las ideas encontradas por Guttmann y Broudo (1989) y Morgado et al., (2009) en el profesorado en ejercicio. No obstante, no podemos olvidar que, además de la propia idiosincrasia de la modalidad y estructura familiar de la que hablemos, su dinámica y clima relacional son variables que determinan la adaptación de sus miembros ( $\underline{\mathrm{Go}}$ lombok, 2006).

Pues bien, aunque se aprecia una visión más positiva hacia la diversidad familiar, consideramos que es necesario invertir mayores esfuerzos de formación del profesorado para derribar prejuicios y desarrollar mayor conocimiento sobre dichas realidades (Maney y Cain, 1997). Valdemoros-San-Emeterio y Lucas-Molina (2014) destacan que el joven alumnado egresado de Educación Primaria reconoce la relevancia de colaborar con la familia. Este dato es esperanzador, pero, además, la formación también les debe permitir aceptar la diversidad familiar a través de una profunda reflexión sobre sus retos específicos (Jones y Blendiger, 1994; López et al., 2008). En nuestra opinión, la formación actual del profesorado resulta aún insuficiente para abordar la complejidad familiar en las aulas. Aunque también, el profesorado debe asumir un compromiso de revisión de sus creencias y prácticas docentes, para fortalecer su propio cambio y hacer realidad la inclusión del alumnado al margen de su familia de procedencia (García-Pérez et al., 2011). Esta meta no puede quedar relegada al albur de la buena voluntad del profesorado, por ejemplo, por la mayor sensibilización de quienes viven circunstancias parecidas (Green y Schaefer, 1985; Rodríguez-Mena y Moreno, 2017). Además, si las familias perciben mayor apoyo y aceptación, quizás también opten por una comunicación más abierta que permitirá acciones más certeras y coordinadas.

Para finalizar, es imperativo comentar que este trabajo sólo supone una exploración general de las expectativas sobre la diversidad familiar del futuro profesorado de Educación Infantil y Primaria, para comprobar si las nuevas generaciones presentan posturas menos prejuiciosas respecto a la diversidad familiar. Ello no es óbice para reconocer que se necesitan más estudios, y más profundos, tanto con este profesorado en formación como con el que está en activo. Por último, es necesario valorar la formación inicial y permanente del profesorado, y comprobar si realmente está contribuyendo a la consecución de estas competencias para su ejercicio profesional.

\section{Referencias Bibliográficas}

Abril, A., y Peinado, M. (2018). Nuevos horizontes familiares: Una reflexión en la formación inicial del profesorado. Profesorado, 22(1), 159-176.

Aguado, L. (2010). Escuela inclusiva y diversidad de modelos familiares. Revista Iberoamericana de Educación, 53(6), 1-11.

Averett, P. E., y Hegde, A. (2012). School social work and early childhood student's attitudes toward gay and lesbian families. Teaching in Higher Education, 17(5), 537-549. doi: https://doi.org/10.1080/13562517.2012.658564

Ball, D. W., Newman, J. M., \& Scheuren, W. J. (1984). Teacher's generalized expectations of children of divorce. Psychological Reports, 54, 347-353. doi: https://doi.org/10.2466/ pr0.1984.54.2.347

Baum, N. (2004). Coping with "absence-presence": noncustodial fathers' parenting behaviors. American Journal of Orthopsychiatry, 74(3), 316-324. doi: https://doi. org/10.1037/0002-9432.74.3.316

Bliss, G. K., \& Harris, M. B. (1999). Teachers' views of students with gay or lesbian parents. Journal of Gay, Lesbian and Bisexual Identity, 4(2), 149-171. 
Bjorklund, D. F., \& Jordan, A. C. (2013). Human parenting from an evolutionary perspective. En W. Bradford, \& K. Kovner, (Coords.), Gender and Parenthood (pp. 61-90). New York: Columbia University Press.

Berástegui, A. (2005). La adaptación familiar en adopción internacional: Una muestra de adoptados mayores de tres años en la Comunidad de Madrid. Madrid: Consejo Económico y Social. Comunidad de Madrid.

Brabender, V. M., \& Fallon, A. E. (2013). Working with Adoptive Parents. New Jersey: John Wiley \& Sons.

Braver, S. L., Ellman, I. M., \& Fabricius, W. V. (2003). Relocation of children after divorce and children's best interests: new evidence and legal considerations. Journal of Family Psychology, 17(2), 206-219. doi: http://dx.doi.org/10.1037/08933200.17.2.206

Cantón, J., y Justicia, M. D. (2007). Características del niño y adaptación al divorcio de los padres. En J. Cantón, M. R. Cortés, y M. D. Justicia (Eds.), Conflictos entre los padres, divorcio y desarrollo de los hijos (pp. 115-132). Madrid: Ediciones Pirámide.

Casper, V., Schultz, S., \& Wickens, E. (1992). Breaking the silences: Lesbian and gay parents and the schools. Teachers College Record, 94(1), 109-137.

Ceballos, M. (2009). La educación formal de los hijos e hijas de familias homoparentales: familia y escuela a contracorriente. Aula Abierta, 37(1), 67-78.

Cortina, C. (2016). Demografía de las parejas homosexuales en España. Revista Española de Investigaciones Sociológicas, 153, 3-22. doi: https://doi.org/10.5477/cis/reis.153.3

Erera, P. I. (2002). Family Diversity. Continuity and change in the contemporary Families. California: Sage Publications.

Farr, R. H., \& Patterson, C.J. (2013). Coparenting among lesbian, gay and heterosexual couples: Associations with adopted children's outcomes. Child Development, 84(4), 1226-1240. doi: https://doi.org/10.1111/cdev.12046

Fredericks, A. D., Rasinsky, T. V., \& Ritty, J. M. (1991). Single-parent family: Tips for educators. The Reading Teacher, 44(8), 604-606.

García-Pérez, R., Rebollo, M. A., Vega, L., Barragán-Sanchez, R., Buzón, O., y Piedra, J. (2011). El patriarcado no es transparente: competencias del profesorado para reconocer desigualdad. Cultura y Educación, 23(3), 385-397. doi: https://doi. org/10.1174/113564011797330298

Ganong, L. H., \& Coleman, M. (2004). Stepfamily relationships: Development, Dynamics, and Interventions. New York: Kluwer Academic.

Golombok, S. (2006). Modelos de familia. Barcelona: Graó.

Golombok, S. (2015). Modern Families. Parents and Children in New Family Forms. United Kingdom: Cambridge University Press.

Gomariz, M. A., Hernández-Prados, M. A., García-Sanz, M. P., y Parra, J. (2017). Tejiendo puentes entre la escuela y la familia. El papel del profesorado. Bordón. Revista de Pedagogía, 69(2), 41-57. doi: https://doi.org/10.13042/Bordon.2016.49832

González, M. M. (2009). Nuevas familias, nuevos retos para la investigación y la educación. Cultura y Educación, 21(4), $381-$ 390. doi: https://doi.org/10.1174/113564009790002409

Green, V. P., \& Schaefer, L. (1985). The effects of personal divorce experience on teacher perceptions of children of divorce. Journal of Divorce, 8(2), 107-110. doi: https://doi.org/10.1300/ J279v08n02_07

Guttmann, J., \& Broudo, M. (1989). The effect of children's family type on children's stereotypes. Journal of Divorce, 12(2-3), 315328. doi: https://doi.org/10.1300/J279v12n02_18

Guttmann, J., Geva, N., \& Gefen, S. (1988). Teachers' and school children's stereotypic perception of "The child divorce".
American Educational Research Journal, 25(4), 555-57. doi: https://doi.org/10.1080/10502550801973096

Hayman, S. (2008). Teach Yourself. Successful step-parenting. Goal a happy family. UK: Hodder Education.

Herbstrith, J. C., Tobin, R. M., Hesson-McInnis, M. S., \& Schneider, W. J. (2013). Preservice teacher attitudes toward gay and lesbian parents. School Psychology Quarterly, 28(3), 183-194. doi: 10.1037/spq0000022

Hetherington, E. M., y Kelly, J. (2005). En lo bueno y en lo malo: la experiencia del divorcio. Cómo influye realmente la separación en la vida de padres e hijos. Barcelona: Paidós.

INE (2018). Encuesta continua de hogares. Año 2017. Nota de prensa de 12 de abril de 2018. Recuperado de http://www. ine.es/prensa/ech_2017.pdf

Jones, L. T., \& Blendiger, J. (1994). New beginnings: preparing future teachers to work with diverse families. Action in teacher education, 16(3), 79-86.

Justicia, M. D., y Cantón, J. (2007). Problemas de adaptación de los hijos divorciados. En J. Cantón, M. R. Cortés, y M. D. Justicia (Eds.), Conflictos entre los padres, divorcio y desarrollo de los hijos (pp. 93-113). Madrid: Ediciones Pirámide.

Loizaga, F. (Coord.) (2010). Adopción hoy. Nuevos desafíos, nuevas estrategias. Bilbao: Ediciones Mensajero.

LOE (2006). Ley Orgánica 2/2006, de 3 de mayo, de Educación. BOE núm. 106, de 4 de mayo de 2006.

LOMCE (2013). Ley Orgánica 8/2013, de 9 de diciembre, para la mejora de la calidad educativa. BOE núm. 295, de 10 de diciembre de 2013.

López, F., Díez, M., Morgado, B., y González, M. M. (2008). Educación infantil y diversidad familiar. XXI. Revista de Educación, 10, 111-122.

Mallon, G. P. (2000). Gay men and lesbians as adoptive parents. Journal of Gay E Lesbian Social Services, 11(4), 1-21. doi: https:// doi.org/10.1300/J041v11n04_01

Maney, D. W., \& Cain, R. E. (1997). Preservice elementary teachers' attitudes toward gay and lesbian parenting. The Journal of School Health, 67(6), 236-241. doi: https://doi. org/10.1111/j.1746-1561.1997.tb06313.x

Marí-Klose, M., y Marí-Klose, P. (2010). Las nuevas modalidades familiares como contexto de transición a la vida adulta: el logro educativo en hogares monoparentales. Juventud y familia desde una perspectiva comparada europea. Revista de Estudios de Juventud, 90, 161-179. Recuperado de: http://hdl. handle.net/2445/56903

MECD (2014). La participación de las familias en la educación escolar. Mirando al futuro. Consejo Escolar del Estado. Madrid: Secretaría General Técnica.

Morgado, B., Jiménez-Lagares, I. y González, M. M. (2009). Ideas del profesorado de primaria acerca de la diversidad familiar. Cultura y Educación, 21(4), 441-451. doi: https://doi. org/10.1174/113564009790002373

Muller, D. (2006). Family-school partnership project. A qualitative and quantitative study. Barton: Australian Government Department of Education, Science and Training.

Oliva, A., y Palacios, J. (2005). Familia y escuela: padres y profesores. En M. J. Rodrigo y J. Palacios (Coords.), Familia y desarrollo humano (pp. 333-350). Madrid: Alianza Editorial.

ORDEN ECI/3857/2007, de 27 de diciembre, por la que se establecen los requisitos para la verificación de los títulos universitarios oficiales que habiliten para el ejercicio de la profesión de Maestro en Educación Primaria. BOE núm. 312, de 29 de diciembre de 2007. MEC.

ORDEN ECI/3854/2007, de 27 de diciembre, por la que se establecen los requisitos para la verificación de los títulos 
universitarios oficiales que habiliten para el ejercicio de la profesión de Maestro en Educación Infantil. BOE núm. 312, de 29 de diciembre de 2007. MEC.

Palacios, J., y Sánchez, Y. (1996). Niños adoptados y no adoptados: un estudio comparativo. Anuario de Psicología, 71, 63-85.

Pryor, J. (2014). Stepfamilies: A Global Perspective on Research, Policy and Practice. New York: Routledge.

Rodrigo, M. J. (Coord.) (2018). Situación de la Infancia y la Familia en Canarias. Madrid: Wolters Kluwer.

Rodríguez-Mena, J. A., \& Moreno, E. (2017). Barriers and limitations to school intervention in family diversity. A case study of homoparental families. Procedia-Social and Behavioral Sciences, 237, 850-855. doi: https://doi.org/10.1016/j.sbspro.2017.02.182

Rodríguez-Ruiz, B., Martínez-González, R. A., y Rodrigo, M. J. (2016). Dificultades de las Familias para Participar en los Centros Escolares. Revista Latinoamericana de Educación Inclusiva, 10(1), 79-98. Recuperado de https://dialnet.unirioja.es/ servlet/articulo? codigo $=5505136$
Sagarna, G. (2010). La adaptación psicológica de niños y niñas adoptados. En F. Loizaga (Coord.), Adopción Hoy. Nuevos desafíos, nuevas estrategias (pp. 255-277). Bilbao: Mensajero.

Scanzoni, J. (2004). Household diversity: The starting point for healthy families in the new century. En M. Coleman y L. Ganong (Eds.), Handbook of Contemporary Families: Considering the Past, Contemplating the Future (pp. 3-22). Thousand Oaks, CA: Sage.

Strohschein, L. (2005). Parental Divorce and Child Mental Health Trajectories. Journal of Marriage and Family, 67, 1286-1300. doi: https://doi.org/10.1111/j.1741-3737.2005.00217.x

Toro-Alfonso, J., y Varas-Díaz, N. (2004). Los otros: prejuicio y distancia social hacia hombres gay y lesbianas en una muestra de estudiantes de nivel universitario. International Journal of Clinical and Health Psychology, 4(3), 537-551.

Valdemoros-San-Emeterio, M. A., y Lucas-Molina, B. (2014). Competencias que configuran el perfil del docente de Primaria. Análisis de la opinión del alumnado de Grado en Educación Primaria. Aula Abierta, 42, 53-60. doi: https://doi. org/10.1016/S0210-2773(14)70009-5 
